\title{
Simple Distributed Temperature Sensor based on Brillouin Gain Spectrum Analysis
}

\author{
Marc Niklès, Luc Thévenaz, Philippe A. Robert \\ Swiss Federal Institute of Technology, Metrology Laboratory \\ $\mathrm{CH}-1015$ Lausanne, Switzerland
}

Brillouin gain spectrum measurement along an optical fiber has recently gained a lot of interests owing to its potentiality for strain monitoring in installed cables [1] and distributed temperature sensing [2]. This measurement requires two lightwaves propagating in opposite directions through the fiber, since Stimulated Brillouin Scattering (SBS) amplification is possible only in the backward direction. One lightwave pumps the medium and the other acts as a probe signal, experiencing amplification when it lies within the Brillouin gain spectral range. The Brillouin Gain Spectrum (BGS) is downshifted with respect to the pump light frequency by the Brillouin frequency shift $v_{B}$ which takes values from 11.5 to $13 \mathrm{GHz}$ depending on the fiber refractive index profile at a pump wavelength near $1300 \mathrm{~nm}$. This frequency shift is given by $v_{B}=2 n V_{a} / \lambda$, where $V_{a}$ is the acoustic velocity within the fiber, $n$ is the refractive index and $\lambda$ the wavelength of the incident lightwave. The temperature dependence of $v_{B}$ makes SBS suitable for temperature sensing.

Therefore the issue of the sensing technique based on BGS analysis is to measure the BGS at different locations along the sensing fiber, because it gives access to $v_{B}$ and consequently to the temperature. Distributed measurements can be performed using a modified OTDR technique. Most of the methods reported so far use either two distinct laser sources [1,2], causing problems when the fiber ends are remote, or employs a single laser source at the expense of the setup complexity [3].

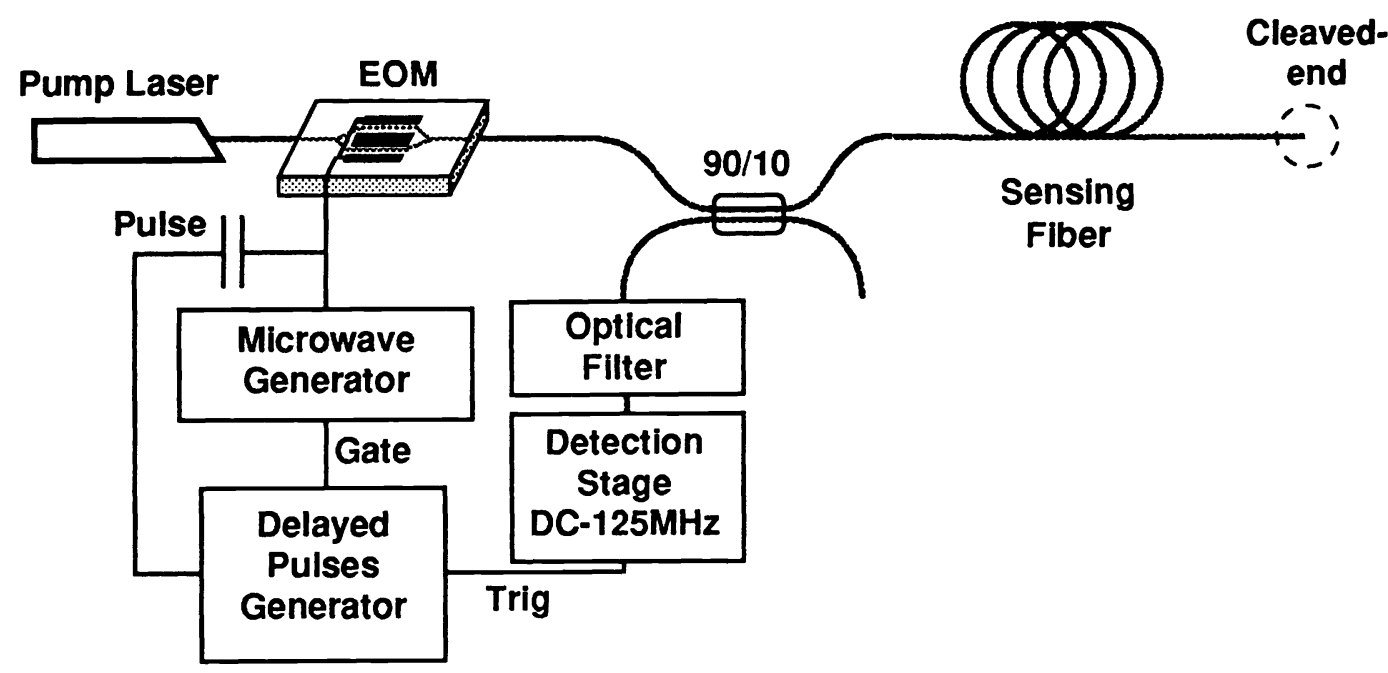

Fig.1: $\quad$ Experimental setup used for distributed temperature measurement.

A novel configuration is presented here, based on the pump and probe technique, that has the following advantages: 1) few optical elements are required, 2) a single laser source is used, 3) a 
single fiber end is accessed, 4) only pulsed signals propagate. A schematic diagram of the experimental setup is shown in Fig.1. The operation of this setup relies on two features [4]. First pump and probe are pulsed signals that both propagate back and forth through the sensing fiber using a reflection at the far end. The pump pulse provides gain to the probe signal during its forward propagation through the SBS process while the probe pulse is amplified on the way back. Therefore the amplification actually only occurs at the crossing point of the pulses, preventing pump power to be depleted before the interaction takes place. Only a few percent of reflection is necessary at the fiber far end, so that Fresnel reflection is actually sufficient.

The second and more important feature is the use of an ultra-wideband integrated $\mathrm{LiNbO}_{3}$ intensity electrooptic modulator (EOM). This modulator is the key element of the setup since it is used for: 1) pulsing the $\mathrm{CW}$ laser light from a $150 \mathrm{~mW}$ Nd:YAG laser to form the pump signal, 2) the generation and frequency tuning of the probe signal. The frequency shift of the laser light is achieved by simply applying a microwave signal on the EOM electrodes. This creates sidebands in the laser spectrum, so that the first lower sideband lies in the BGS generated by the pump, when the modulation frequency $f_{m}$ is close to the Brillouin frequency shift $v_{B}$ [5]. The first upper sideband and higher order sidebands are optically filtered out by a grating. This technique makes the scan of the probe optical frequency very simple and convenient by just varying the microwave signal frequency. In addition the probe can be simply pulsed by gating the microwave generator output. The DC bias setting on the EOM does not change the sidebands' amplitude and just determines the amount of transmitted amplitude of the fundamental frequency, as shown in Fig.2.
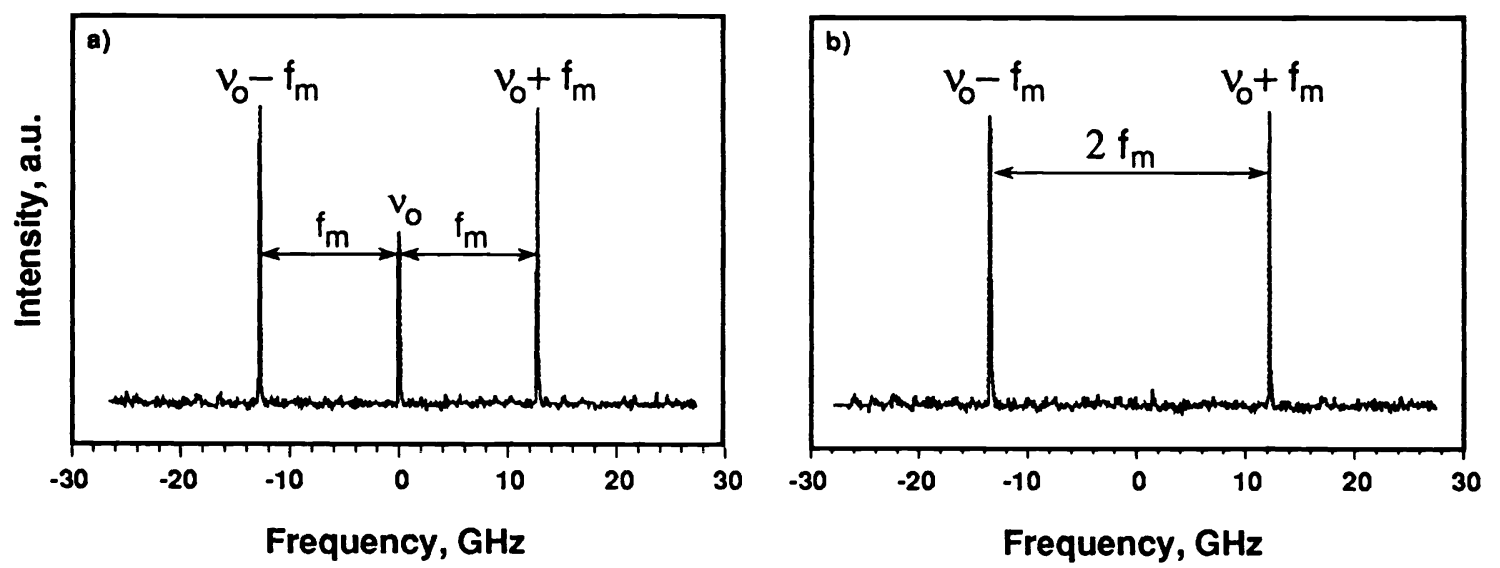

Fig.2: $\quad$ Optical intensity spectra of a single frequency laser modulated by an electrooptic modulator scanned with a Fabry-Perot analyzer a) Carrier and first order sidebands b) Suppression of the carrier by setting the proper DC-bias on the modulator electrodes.

Figure 3 shows the transfer function of the EOM and the timing of the electrical driving signals applied to the EOM electrodes. The DC-bias is first set so that no light goes through the EOM. At time $T_{0}$ and for a short time, the EOM modulates the laser light at frequency $f_{m}$, in order to generate a probe pulse with optical spectrum identical to Fig.2b. At time $T_{1}$ the modulator is switched on by applying an electrical pulse on the EOM electrodes, creating a pump pulse at $v_{0}$. A digital delay 
generator is used to adjust the delay $\Delta T=T_{1}-T_{0}$ between the probe and pump pulses, so that they cross at a definite location. Every positions along the sensing fiber can be addressed this way.

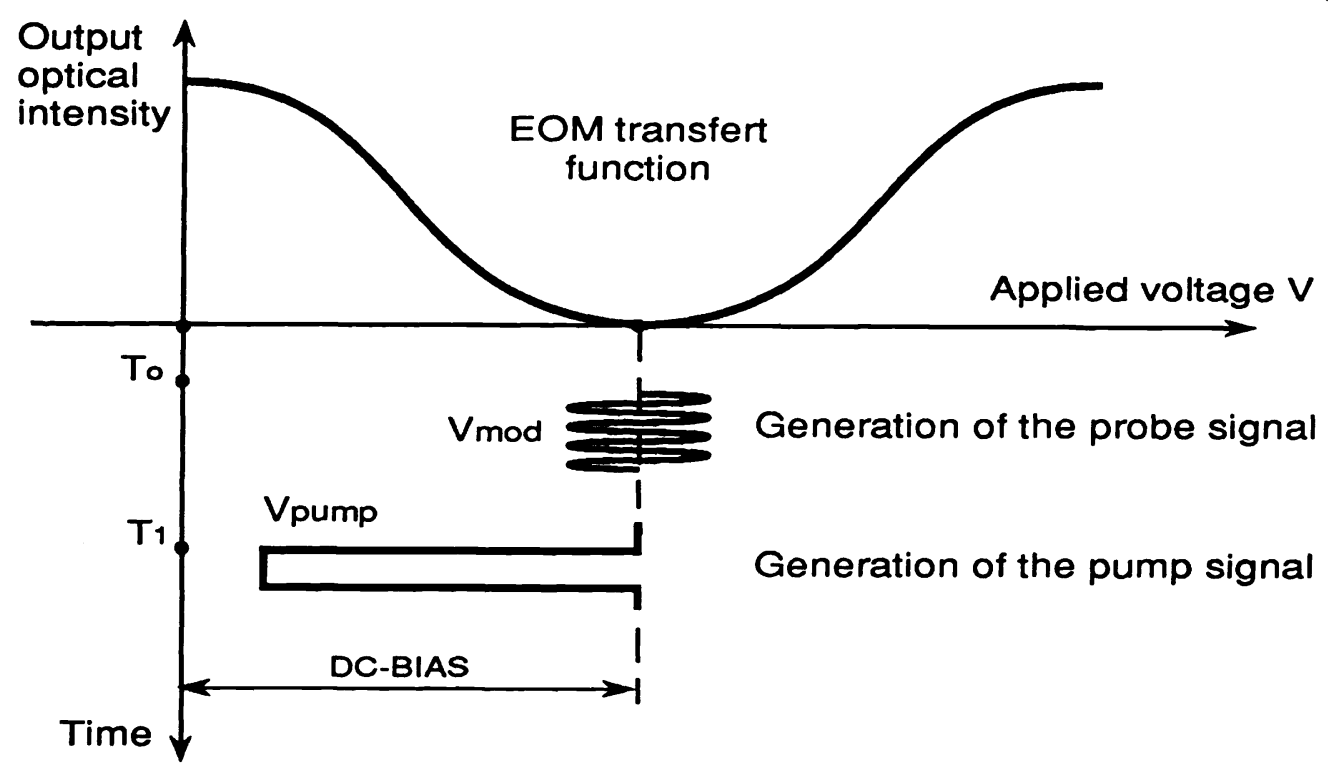

Fig.3: $\quad$ Schematic diagram of the EOM transfer function and the electrical driving signals

Since this technique uses a single laser and a modulator for the generation of the pump and probe signals, it insures an inherent stability of their frequency difference. Measurements of the distribution of the Brillouin frequency shift along a sensing fiber can therefore be performed with an excellent resolution using this technique. The measured standard deviation in the determination of the Brillouin shift frequency is $300 \mathrm{kHz}$. The best spatial resolution obtained so far is $60 \mathrm{~m}$, given by the minimum pump pulse width leaving a sufficient gain.

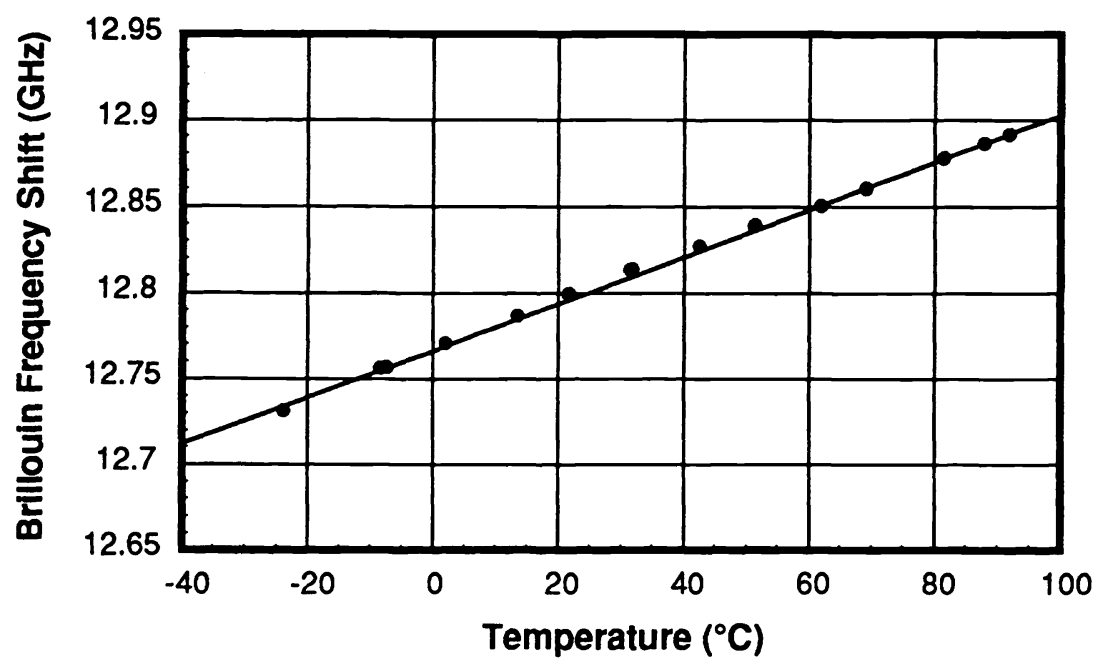

Fig.4: $\quad$ Temperature dependence of the Brillouin frequency shift for a standard single mode fiber.

The temperature dependence of $v_{B}$ is represented in Fig.4. For a standard bare fiber the coefficient of the frequency shift change is found to be $1.36 \mathrm{MHz} /{ }^{\circ} \mathrm{C}$. Consequently the temperature resolution is better than $0.5^{\circ} \mathrm{C}$. Fig. 5 shows the distribution of the Brillouin frequency shift along $4.7 \mathrm{~km}$ fiber, 
measured using the setup in Fig.1. This fiber is actually made up of three segments of fiber spliced together. These fibers (coming from the same production, but not from the same preform) are wound on three different coils. The distributed measurement of $v_{B}$ was done twice: A) the first fiber (1.5km) was placed in an oven and brought to the temperature of $55^{\circ} \mathrm{C}$, fibers 2 and 3 at room temperature, B) fiber 1, 2 and 3 were at room temperature. Measurement $B$ shows that the 3 fibers present a slight different $v_{B}$ even though they are at the same temperature. When the first fiber is heated the change in the Brillouin frequency shift can be easily measured and the temperature elevation can be determined using the calibration measurement shown in Fig.4.

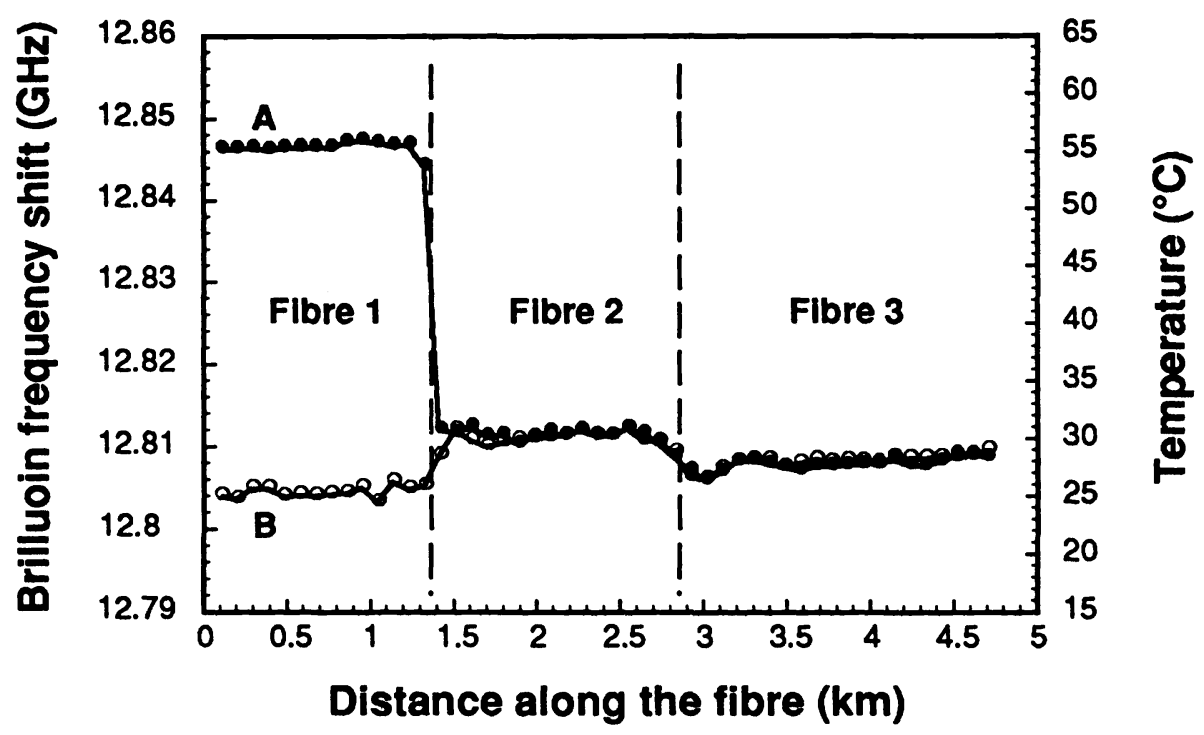

Fig.5: Distributed measurement of the Brillouin frequency shift (and the equivalence in terms of temperature) for 3 fibers spliced together. A) Fiber 1 placed in an oven and brought to the temperature of $55^{\circ} \mathrm{C}$, fiber 2 and 3 at room temperature B) Fiber 1,2 and 3 at room temperature

A new configuration for distributed temperature measurement based on Brillouin gain spectrum analysis has been developed. It presents the main advantage to require few optical elements and only one laser source and shows a great inherent stability, consequently. It is therefore very promising for the further development of an instrument. The results from this technique are steadily improving along with system optimization, in particular for spatial resolution.

Acknowledgments: the authors are grateful to the Swiss PTT and PSEL funding for their financial support and valuable discussions.

\section{References}

1. T.Horiguchi, T.Kurashima, M.Tateda, IEEE Photonics Technology Letters, 2, (1990), pp.352-354.

2. X.Bao, D.J.Webb, D.A.Jackson, Optics Letters, 18, (1993), pp.552-554.

3. K.Shimizu, T.Horiguchi, Y.Koyamada, T.Kurashima, Optics Letters, 18, (1993), pp.185-187.

4. M.Niklès, L.Thévenaz, P.Robert, Proceedings of the Conference on Optical Fiber Communications OFC'94, OSA Publications, paper WF1, pp.89 (1994).

5. M.Niklès, L.Thévenaz, P.Robert, Proceedings of the Conference on Nonlinear GuidedWave Phenomena, OSA Publications, pp.92 (1993). 